\title{
Applications of geoinformation technologies in the grain farming industry
}

\author{
Svetlana Petrova and Danil Zyukin \\ Kursk State Agricultural Academy, 70, K.Marx street, Kursk, 305021,Russia
}

\begin{abstract}
The paper studies the introduction and the most effective use of information technologies in grain economy, the largest and most important segment of the Russian agro-industrial complex. The state of this segment strongly determines the food security and the development of related agricultural areas. Information technology is presented as a fundamental factor in the increase of the competitiveness of national grain in global market, as it ensures cost optimization at all stages of the reproduction chain of the grain-product sub-complex of the agro-industrial complex: from the cultivation of grain crops to logistics operations and grain delivery to international partners. There are many grain production automation areas. Geoinformation technologies are the most effective and rapidly developing area. The article examines modules that can be combined into a single information system that can be used by grain producers or as independent software systems. Within each direction, attention is paid to each area. The article aims to find the most efficient and less costly ways to increase the volume of grain production. The study found out that the automation of even one area can increase the turnover in grain production. However, this method is not less expensive. It requires various resources: financial, technical, time, and labour ones.
\end{abstract}

\section{Introduction}

For a long time, large-scale innovative projects intended for the development of the agricultural sector faced high investment risks due to the long and labor-intensive production cycle. The success of projects depends on natural conditions, organizational and production factors that determine shortcomings in the cultivation, harvesting, transportation and storage of grain. The most popular solutions to these problems are impacts on the external conditions through technological solutions.

For a long time, the Russian agricultural industry was not automated, projects designed to implement information technologies into biological processes were not funded. This caused the lag of Russia in the productivity and technical equipment.

Currently, the low level of automation and technical equipment affects the competitiveness of products in the world market. In Russia, wheat production has the greatest export potential [1].

In the conditions of full satisfaction of national market, it is export that is the main motivation to increase gross receipts in Russia. However, to achieve this, exports must remain consistently profitable, regardless of the situation on the world grain market. The main way to ensure the competitiveness and efficiency of exports is to optimize costs, which can be achieved at all stages of the cycle: from landing to cargo transportation to final international buyers. In the context of a relatively high degree of intensification of the cultivation of grain crops in the main grain-producing regions of the country, it is difficult to achieve cost reduction by means of efficiency increase. Therefore, in our research, we consider the development of the production and logistics infrastructure of the grainproduct sub-complex of the agro-industrial complex as a priority [2].

Innovative technologies are involved both in the process of direct cultivation of grain crops and in the transportation of grain.

To ensure the high efficiency of grain production, the well-developed technical base is required. The most relevant areas of automation and digitalization are grain harvesting, transportation and storage.

\section{Results and Discussion}

\subsection{Problems of using modern automated tools in the grain industry}

The grain production is a set of actions aimed at obtaining high-quality raw materials. This process should be controlled. The use of automated tools at other stages is also important for achieving the targets.

Application of technological achievements is accompanied by an increase in the labor productivity in all the agricultural areas [3]. Their effective and rational application determines a set of tasks: funding the implementation of new technologies. For example, machines require large financial investments and maintenance and storage spaces.

The digital technologies can reduce costs, simplify production and organizational processes. Nevertheless, these projects are still not easy to implement and integrate into the production cycle in all agricultural

\footnotetext{
Corresponding author: svet-orl@yandex.ru
} 
areas, including grain farming. The information systems were used to solve planning, financing, reporting, and logistic problems.

Any production activity is influenced by many factors that determine its constant change in order to meet modern requirements and market needs. To increase the volume of grain production, a new leap in the development of technologies is required. Digital technology allows for the "smart" monitoring of all grain production processes. Areas such as the artificial intelligence, the decision-making theory, the machine learning have created a large number of different analogs using a wide range of digital technologies.

\subsection{Applications of geoinformation technologies in the grain production industry}

Every sphere of human life is constantly growing and developing. Thus, the tasks of grain production are characterized by a constant search for opportunities to improve quality, gross harvest and ensure efficiency increase. The last aspect in a market economy is an integral development criterion and it is achieved in completely different ways. For example, in order to solve the issues of financing, accounting and logistics, they often use information systems, since it becomes possible to increase their effectiveness several times due to the development of related processes [4].

The structure of any information system is based on the work with data, such as storage, exchange and analysis. Without a well-established link between core activities and the system, the entire software structure is virtually uncontrollable. All actions take place outside the scope of the program. At the same time, the information system must receive initial data on the results of each ongoing process.

Previously, at the initial stages of automation of agricultural production processes, the intervention of system operators in obtaining data was used. It is necessary to note that according to the trends of the modern world, the concept of automation has acquired a different meaning. Thus, previously, an activity was considered automated if machines were involved in the process. Today, tracking by the data synchronization system is no longer a sufficient condition for the successful development of production. All necessary variables that are used in software modules must be obtained automatically, that is, using special devices and sensors. Only in this case can we talk about significant changes in the efficiency of implementation of software systems [5].

Without the introduction and use of special electronics, information enters the system through the manual input of operators, which increases the possibility of further paperwork. In this case, there is a high probability of various errors occurring when filling out the necessary forms of the information system, data loss or deliberate distortion, which is unacceptable in the achievement of the strategic goals of production development. In accordance with this, it becomes necessary to use other methods based on a combination of software and hardware.

Among information technologies used in the grain production industry, there are geoinformation technologies. The use of information systems with a business architecture based on these principles can increase the productivity due to the following functions:

- automatic decision-making;

- improvement of technologies for planning agrotechnical activities;

- monitoring of the main activities within the production life cycle: tracking parallel states at different stages, analyzing intermediate results, losses and profit, tracking the quality of raw materials;

- forecasting the yield depending on various factors;

- monitoring the use of resources [6].

Despite these advantages of digital technologies, it is not possible to abandon the use of traditional tools. However, the combined use of traditional and new technologies can be beneficial.

\subsection{Decision-making systems for managing key processes}

The decision-making system takes over the management of key processes, which are constituent elements of each stage. It uses a multi-layer map of the grain farming process and a database containing required data. The main data are as follows:

- tables describing the main characteristics of the territory;

- information about the territory obtained by means of remote sensing;

- data on crop properties;

- data on soil properties;

- crop maps;

- data on the work carried out.

When designing the database, the traditional database architecture building methods based on the relational model (SQL) or the method of non-relational interpretation of storage tools can be used. Both technologies have a number of advantages and disadvantages. The relational models are more widespread technologies. At the same time, this interpretation of data that has a complex structure and can cause losses of information resources (the memory volume and information processing time). The simplest solution is to use non-relational databases (NoSQL). Their architecture is simpler and cheaper. However, there are few specialists capable of working with this system. The expediency of using one or another model of data interpretation depends on the capabilities of grain production enterprises. Large-scale enterprises having required specialists can expand functions of the existing data interpretation tools rather than implement new ones. This will help to prevent problems of data transfer and conversion.

These characteristics are linked to the electronic map based on an external database language, which describes the interaction of the digital terrain map application with the data warehouse. Conventionally, this link can be 
represented as a multilayer object. The first level is a hydrographic network. It can be supplemented with other characteristics. On other layers, there is a link with the data characterizing the soil and sowing areas.

The automated control system allows for a complete analysis of areas through the use of geodetic research methods. These principles allow for the detailed study of large-scale areas.

Remote sensing capabilities transmit up-to-date information about grain production processes and soil and crop characteristics. This is the main advantage of the decision-making support information system.

The design of modules that automate the decisionmaking process in geographic information systems requires a special approach. The tasks set for the program can be classified as intellectual, which requires a special individual approach. In the traditional practice of informatics and computer technology, computers, as a rule, operate according to a certain algorithm, performing a limited and previously implemented order of actions. In problem solution, decision-making requires an individual approach, which today does not yet have an optimal solution. The information system is faced with the most difficult task of coming up with this solution.

During the designing of decision-making systems, it is necessary to follow the following principles:

- The simplification of interaction processes between different production departments, software models;

- The simplification of information exchange processes between various categories of specialists, hardware devices, programs;

- The provision of complete information about the type of activity, however, the transmitted and received parameters should not be excessive. Otherwise, it will be difficult to process and understand them by the users of the system.

The existing decision-making systems can not completely replace humans. On the one hand, they already perform a fairly wide range of functions, but a technician must constantly monitor the quality of work. At the same time, now the developers do not face the task of the creation of a universal system that could completely replace an expert in grain production. The highest priority goal is to find the ways of effective interaction of common human efforts and hardware and software tools.

\subsection{Systems for planning agricultural activities and monitoring the activities within the production life cycle}

Systems for planning agricultural activities are one more area of geographic information platforms. These types of measures are important for improving grain production conditions. Therefore, it is important to implement optimal planning measures. When implementing information systems, the following technologies are used:

- personnel recruiting, distribution of tasks in accordance with skills;
- measurement of the working space using digital devices and information systems;

- design of the crop space using a vector model;

- calculation and assessment of the funds (fertilizers, plant protection products, growth regulators, soil cultivation equipment);

- planning of grain production processes taking into account the above parameters (personnel, time, equipment, raw materials);

- control and analysis of results.

The use of software systems allows you to collect complete information about all processes and draw up a detailed plan of each work. Modern information products can calculate the timing with an accuracy of up to hours. However, when choosing a technology, it is advisable to set the day as the smallest calculated time unit. Otherwise, the probability of difficulties is not taken into account, or it is taken into account, but the equipment downtime can occur. This has a certain psychological burden, which affects the performance of workers. Control exercised by a person describes the methods of managing organizational behavior that must be taken into account when setting up or designing information systems.

At the current stage of program development, there are unified services, designed and customized for the needs of each organization in accordance with its industry specifics (e.g., Cropio, OneSoil, ExactFarming, etc.). It is worth integrating new technologies such as receiving data from new digital devices, cloud storage of data, communication within the organization and with external departments.

The automation of agrotechnical processes reduces the waste of resources, helps to distribute tasks, identify and describe problems to decrease the cost of resources required.

The main activities within the production cycle are monitored (expert estimates and forecasts, data storage). It is possible to assess the situation by using the artificial intelligence technology to draw conclusions based on the data and offer solutions to the problems). These technologies are not common in other industries.

\subsection{Forecasting systems}

The forecasting is crucial in organizing activities of agricultural producers. It involves an analysis of conditions affecting the grain production, the grain market and needs of the end consumer. It allows you to set final goals, identify production efficiency criteria and use this data for planning agricultural processes. The results are used as a reference or model value to assess the efficiency of the decision made and activities performed [7].

Information systems used for forecasting are based on the methods of observation of the state of crops under various weather conditions. It is important to track the dynamics of crop development and growing conditions.

The system allows you to make economic forecasts. It is important to with this system, compare the results 
with the benchmark, enter real data and improve the forecasts made.

The complexity of this system is due to a combination of various data, their recording and storage. It is difficult to distribute mixed data flows. This operation is performed by building a data model architecture based on objects and their attributes. This model includes various objects: layers, tables, graphs, drawings, terrain images obtained using various digital means.

\subsection{Resource Usage Monitoring}

The system monitoring the use of resources is based on the results of planning and divides the operations into several flows in accordance with the type of resources. All functions can be divided into several subsystems. When designing a business model, opportunities are aggregated into separate blocks. For the user, the interface is grouped links to specific objects that describe a specific operation (tables, lists, fields for input and output of data, buttons for confirming or canceling transactions). In accordance with the level of an employee, you can define the configuration of access roles to certain data, which allows you to use a single system and a single interface and avoid problems in the design of duplicates with different purposes.

The following tasks can be distributed according to the type of resources:

- monitoring of equipment;

- monitoring of the workload of personnel;

- monitoring of costs of auxiliary raw materials (fertilizers, plant protection products).

It is difficult to control the use of agricultural machinery (e.g., repair planning). This function is used only when required. However, planning is a must. This process allows you to determine the time of downtime or overload in order to avoid financial losses. The machines should be examined regularly. Scheduled inspections are recorded in the schedule. A malfunction that can affect the operation of the machine may be identified. It will be necessary to make changes to the existing plan, replace equipment and assign personnel for repairs. Therefore, it is important to develop flexible schedules.

Monitoring of technical devices solves logistics problems. The system determines optimal routes, their duration and wear of equipment, allows you to control the speed of delivery of raw materials and products, and generates reports for various parameters.

The role of the labor factor is crucial; the technical component of this factor is being updated. HR specialists select personnel being responsible for their performance, and monitor the performance of each employee. There are systems that monitor employee performance. In many industries, automated workstations have been implemented. They provide employees with access to the system to obtain information and upload their own information. The functions are determined by specializations. In the information system, this is reflected as a role - a set of privileges or permissions, what is permissible for the user to perform and what is prohibited. This is reflected through the interface. Functions for which the user does not have permission are not displayed in his interface. For different categories of personnel, different devices are used to access the system. For office workers, the most convenient format is a personal computer. For other categories of employees who often change locations, the most convenient device is a smartphone. These tools should be used to prevent unauthorized access to the system.

Reports on works performed are uploaded to the system by employees. On the one hand, it incurs costs for the purchase of electronic devices; on the other hand, it increases the role of monitoring. Geoinformation technologies are used to receive data entered by employees and monitor their locations, track the duration of their activities.

\section{Conclusion}

Geoinformation technologies make it possible to solve problems arising in the grain farming industry. Due to these technologies, it is possible to automate a large number of processes that affect the productivity of industries.

The most expensive equipment is hardware tools. The technologies are expensive due to the economic conditions in Russia. In addition, it is expensive and problematic to cover all areas of automation in one system due to the complex nature of architecture modeling and design of communication channels. Moreover, to create the system and adapt it to real operating conditions is time-consuming and costly. With all the prospects for the innovative development of agriculture, the labor factor is a problem which hinders the optimal use of all advantages of the technology.

\section{References}

1. D.A. Zyukin, O.N. Pronskaya, O.V. Svyatova, A.A. Golovin, O.V. Pshenichnikova, O.V. Petrushina Revista de la universidad del Zulia. 32, 87-101, (2021).

2. D. Zyukin, O. Svyatova, E. Zolotareva, A. Bystritskaya, A. Alyokhina Amazonia Investiga, 9(25), 461-470, (2020).

3. J. Geng, X. Wang, S. Zhu, X. Lin The Journal of Engeneering 15, 547-551, (2019).

4. L. Oveshnikova, O. Lebedinskaya, A. Timofeev, L. Mikheykina, E. Sibirskaya, P. Lula Advances in Intelligent Systems and Computing. 726, 87-96 (2019).

5. O.G. Lebedinskaya, A.G. Timofeev, E.A. Yarnykh, N.A. Eldyaeva, S.V. Golodov Advances in Intelligent Systems and Computing. 622, 510518, (2018).

6. S. R. Swaffield, R. C. Corry, P. Opdam, W. Mc. William, J. Primdahl Business Strategy and the Enviroment 28 (7), 1357-1369, (2019) 
7. L. Ran, Y. Yuan, E. Cooter, V. Benson, D. Yang, J. Pleim, R. Wang, J. Williams Journal of Advances in Modeling Earth System 12(11), 4645-4668,(2019). 